\title{
Exciton-light coupling in quantum wells: From motional narrowing to superradiance
}

\author{
Alexey V. Kavokin* \\ INFM, Dipartimento di Ingeneria e Elettronica, Università di Roma “Tor Vergata," via della Ricerca Scientifica 1, 00133, Roma, Italy \\ Jeremy J. Baumberg \\ Hitachi Cambridge Laboratory, Cavendish Laboratory, Madingley Road, Cambridge, CB3 OHE, United Kingdom
}

(Received 15 December 1997)

\begin{abstract}
We show numerically that spatial delocalization of excition-polariton states in multiple quantum wells leads to efficient narrowing of exciton resonances with respect to the single-quantum-well $(\mathrm{QW})$ case. This is a manifestation of a motional narrowing effect, i.e., averaging of short-range potential fluctuations by extended exciton-polariton modes. Increasing the number of QW's or decreasing the degree of disorder, one recovers the superradiance regime where the exciton linewidth is governed by the splitting between the strongest excitonpolariton modes and now increases with an increasing number of QW's. Vertical disorder, in which the different wells are no longer equivalent, suppresses both effects. [S0163-1829(98)50720-5]
\end{abstract}

Exciton-photon coupling in quantum well structures still elicits significant fundamental interest. Starting from the pioneering work by Agranovich and Dubovskii, ${ }^{1}$ elaborated theories of exciton polaritons in two-dimensional systems have been created both within quantum and semiclassical approaches. Exciton polaritons are quasiparticles appearing due to the exciton-light interaction and having a finite radiative lifetime in two dimensional systems. The difference between exciton polaritons and excitons, to first order, is their different spatial extent: an exciton is localized in a single quantum well $(\mathrm{QW})$ while an exciton polariton propagates through the entire structure.

As will be shown below, this difference may be unavoidable in real systems because of the presence of potential fluctuations necessarily arising from QW width fluctuations, alloy fluctuations, random fields from defects, etc. These fluctuations cause inhomogeneous broadening of exciton resonances (to be distinguished from the homogeneous broadening due to phonons).

The idea of this work is to show that exciton polaritons in multiple quantum wells (MQW's), being extended quantum particles, are subject to the quantum motional narrowing effect. This effect, known in atomic physics, ${ }^{2}$ reduces the width of spectral lines corresponding to some quantum transition due to averaging of the disorder potential in the system within the localization radius of a particle participating in the transition. In our case, the polaritonic motional narrowing is manifest in a reduction of the width of the exciton resonance in MQW's with respect to a single QW having the same distribution function of in-plane potential fluctuations. The exciton-polariton mode averages the fluctuation potential along the structure growth direction, which leads to the narrowing of spectral lines.

Motional narrowing provides longer exciton radiative decay times in typical MQW structures than in single QW's contrary to predictions from existing superradiance theory, which do not take into account the effect of exciton inhomogeneous broadening. ${ }^{3}$ The term "superradiance" in this context means enhancement of the radiative decay rate of excitons in MQW's due to multiple reflections between QW's.
The superradiance regime is realized when the excitonpolariton radiative decay rate exceeds the inhomogeneous broadening and may be achieved by a further increase in the number of QW's in the structure. All the above effects completely disappear if the average exciton resonance frequency changes from well to well because this "vertical disorder" destroys the extended coherence in the system.

Disorder effects on the exciton radiative properties in quantum wells have been extensively studied theoretically and experimentally during recent years. ${ }^{4}$ The closest to our present theoretical work ${ }^{5}$ dealing with time-resolved dynamics of MQW structures, presented a semiclassical technique allowing both disorder and polariton effects to be taken into account. No emphasis was put on disorder averaging or superradiance effects. An accurate quantum-mechanical theory of the potential fluctuation effect on the dielectric susceptibility of quantum wells has been developed by Zimmermann. ${ }^{6}$ This theory, however, does not take into account the retarded light-exciton interaction, i.e., the polariton effect, which is the subject of our present study. In the last two years substantial interest has been focused on the motional narrowing problem in semiconductor microcavities with embedded quantum wells. ${ }^{7,8}$ In this kind of system, the reduction of the width of exciton-polariton resonances at the anticrossing of cavity photon and exciton modes has also been associated with averaging of the fluctuation potential in the QW plane.

This in-plane averaging in a single QW should be distinguished from the vertical averaging in MQW structures that is considered here. It is difficult to unambiguously associate a lateral size with the exciton polaritons because they consist of coherently coupled localized excitons that are separated by distances on the order of the wavelength of resonant light. However, at normal incidence in the MQW's, there is no change to the in-plane dispersion. In contrast, polariton effects in microcavities, according to Ref. 7, lead to the lightening of the exciton in-plane mass that changes the exciton energy distribution function. We also note that the data ${ }^{7}$ can equally be explained without the last assumption. ${ }^{9}$ Finally, 
the first experimental data on the vertical motional narrowing in MQW's have been obtained very recently by Baumberg et al. ${ }^{10}$

In the present work we propose and prove the concept of motional narrowing-superradiance interplay in MQW's. We have extended the recently developed semiclassical theory of exciton inhomogeneous broadening in QW' $\mathrm{s}^{9,11}$ and applied it to our specific problem: the exciton resonance line-shape dependence on the number of QW's in a MQW structure. The approach is based on the solution of Maxwell equations for light incident on the structure taking into account the nonlocal contribution of the inhomogeneously broadened exciton resonance to the dielectric susceptibility of the QW's. Reflectivity of the MQW structures is calculated by a transfer-matrix method with Maxwell boundary conditions.

The transfer matrix across one period of the MQW connecting the amplitudes of light waves propagating forward and backward in the structure is

$$
T=\frac{1}{t}\left[\begin{array}{cc}
\left(t^{2}-r^{2}\right) e^{i k d} & r e^{-i k d} \\
-r e^{i k d} & e^{-i k d}
\end{array}\right],
$$

where $d$ is the period of the structure, $k$ is the wave vector of light in the media, with amplitude reflection and transmission coefficients of the QW

$$
r=\frac{i \alpha \chi}{1-i \alpha \chi}, \quad t=1+r .
$$

Here $\chi$ is the dielectric susceptibility of the quantum well, which contains contributions distributed in frequency from all the exciton resonances through a function $f(\omega)$ due to the exciton inhomogeneous broadening, and $\alpha$ is a parameter proportional to the exciton radiative decay rate. We assume the wave vector of light in the plane of the QW's to be conserved. Although $f(\omega)$ is frequently assumed to be symmetric, a realistic resonance frequency distribution produced by the coupling of light with exciton states in a disorder potential is inevitably asymmetric. ${ }^{12,13}$ This is due to the finite quantum confinement energy of the excitons forming its lower energy tail. A symmetrical distribution introduces pronounced additional destructive interferences in time ${ }^{11}$ that are washed out in experiments due to this asymmetry. In the following we will assume $f(\omega)$ takes the form of an asymmetric Gaussian broadened by a parameter $\Delta_{1}$ at energies lower than $\omega_{0}$ and by $\Delta_{2}$ at energies higher than $\omega_{0}$.

Representing the eigenvalues of the matrix (1) in the form $\lambda_{ \pm}=e^{ \pm i Q d}$ with corresponding eigenvectors $I_{ \pm}=\left[\begin{array}{c}1 \\ a_{ \pm}\end{array}\right]$where $a_{ \pm}=r e^{i k d} / e^{(-i k d}-t e^{ \pm i Q d)}$, one can obtain using standard boundary conditions (no light incident from $\pm \infty$ ) an equation for polariton eigenenergies

$$
a_{+} e^{i N Q d}=a_{-} e^{-i N Q d},
$$

where $N$ is the number of QW's. Solutions of Eq. (3) form a spectrum of so-called "dark' and "bright'" polariton modes (for details see Ref. 3). The parameter governing "darkness", and "brightness" of the mode is the imaginary part of its complex eigenfrequency. The size of this imaginary part is proportional to the oscillator strength of the given mode and inversely proportional to its lifetime. "Bright"' or superradiant modes in MQW's have eigenfrequencies whose imagi-

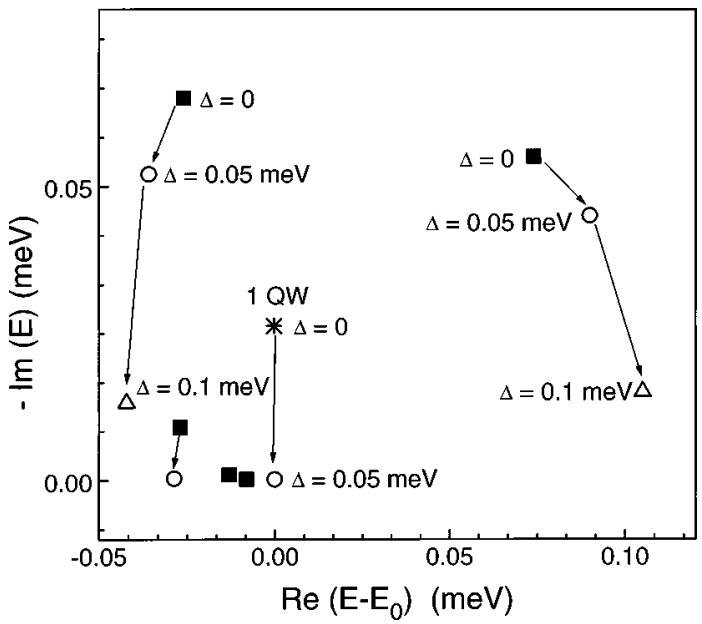

FIG. 1. Complex eigenenergies $\left(E_{i}\right)$ of the exciton-polariton modes in a periodic structure of $5 \mathrm{QW}$ 's described in the text, in the absence (solid squares) and in the presence (circles and triangles) of disorder. For comparison, the exciton-polariton eigenenergy in a single QW is shown in the absence of disorder (star) and at $\Delta=0.05$ $\mathrm{meV}$ (circle). $E_{0}=1.543 \mathrm{eV}$.

nary part exceeds the imaginary part of the single QW polariton mode. "Dark' modes have an imaginary part of the eigenfrequency close to zero.

In the case of no disorder, a sum rule is valid for excitonpolariton eigenfrequencies $\omega_{j}$ :

$$
\sum_{i=1}^{N}\left[\omega_{i}-\omega_{0}+i\left(\gamma+\Gamma_{0}\right)\right]=0
$$

where $\omega_{0}$ is the exciton resonance frequency, $\gamma$ is the exciton homogeneous broadening, and $\Gamma_{0}$ is its radiative decay rate. As follows from Eq. (4), only few very bright modes can be present in the system. Approximate analytical formulas for eigenenergies of dark and bright modes are given in Ref. 14.

Figure 1 shows the complex eigenenergies of exciton polaritons in a stack of five $\mathrm{GaAs} / \mathrm{Al}_{x} \mathrm{Ga}_{1-x} \mathrm{As}$ quantum wells $\left(\hbar \omega_{0}=1.543 \mathrm{eV}, d=240 \AA, \gamma=0, \hbar \Gamma_{0}=0.026 \mathrm{meV}\right)$ for different values of the disorder parameter $\Delta=\Delta_{1}=\Delta_{2}$. In the absence of disorder this structure is characterized by two bright and three dark modes (solid squares). In the presence of disorder, the spectrum of eigenmodes changes drastically, bright modes become much darker while the splitting between them slightly increases. For comparison, the excitonpolariton eigenfrequency in a single QW is also shown.

Clearly, the inhomogeneous broadening must suppress the optical coupling between different wells because disorder destroys the coherence in the system. In order to get a definitive handle on this effect, we have considered Braggarranged MQW's where only one superradiant polariton mode has nonzero oscillator strength. ${ }^{15}$ The dependence with $\Delta$ of the imaginary part of the eigenenergy of this sole polariton mode is shown in Fig. 2. One can see that the superradiant mode in $10 \mathrm{QW}$ 's is much more resistive to disorder than the modes in 5 and 1 QW's. This illustrates the general rule: brighter polariton modes are less influenced by disorder than darker modes. The competition between polaritonic coupling and inhomogeneous broadening in the system is responsible for the motional narrowing effect in MQW's. 


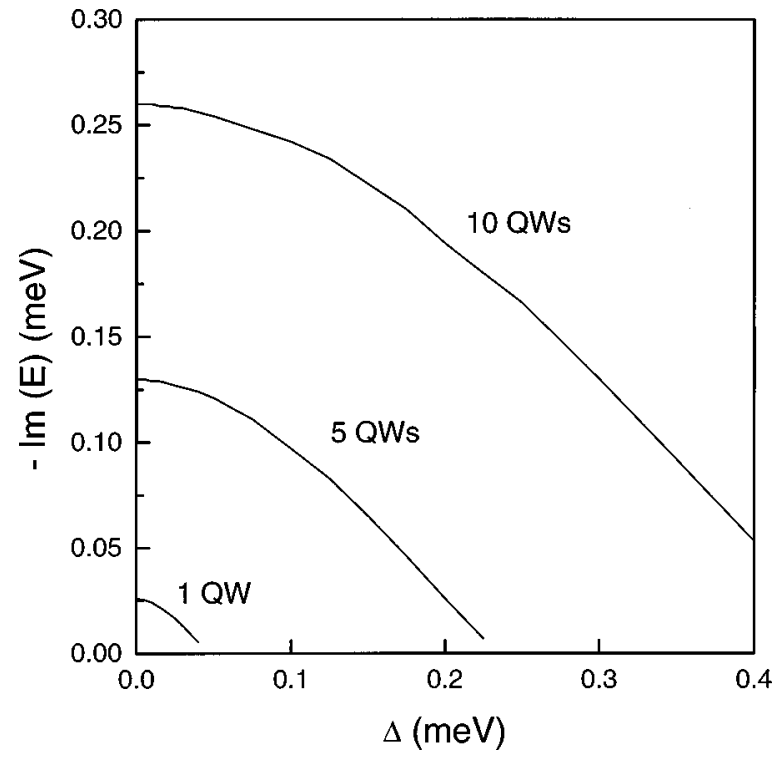

FIG. 2. Imaginary part of the complex eigenenergy of the superradiant mode in 1, 5, and 10 Bragg-arranged QW's vs disorder parameter $\Delta$.

Figure 3 shows the calculated reflection spectra from periodic structures with $1,5,10,25$, and 50 QW's. The parameters $\left(\omega_{0}, d, \gamma, \Gamma_{0}\right)$ for all these structures are the same as before. Asymmetric inhomogeneous distributions of excitons have been adopted with broadenings $\Delta_{1}=0.4 \mathrm{meV}, \Delta_{2}$ $=0.7 \mathrm{meV}$ in order to make the model systems as close as possible to the experimentally studied ones. ${ }^{10}$ The line-shape variations with changes in the number of QW's in the structure are quite remarkable. The linewidth decreases with increase of $N$ from 1 to 10 , then it starts to increase again, and a fine structure of the exciton resonance appears.

The initial decrease of the linewidth with increase $N$ is a direct manifestation of the motional narrowing effect. Ex-

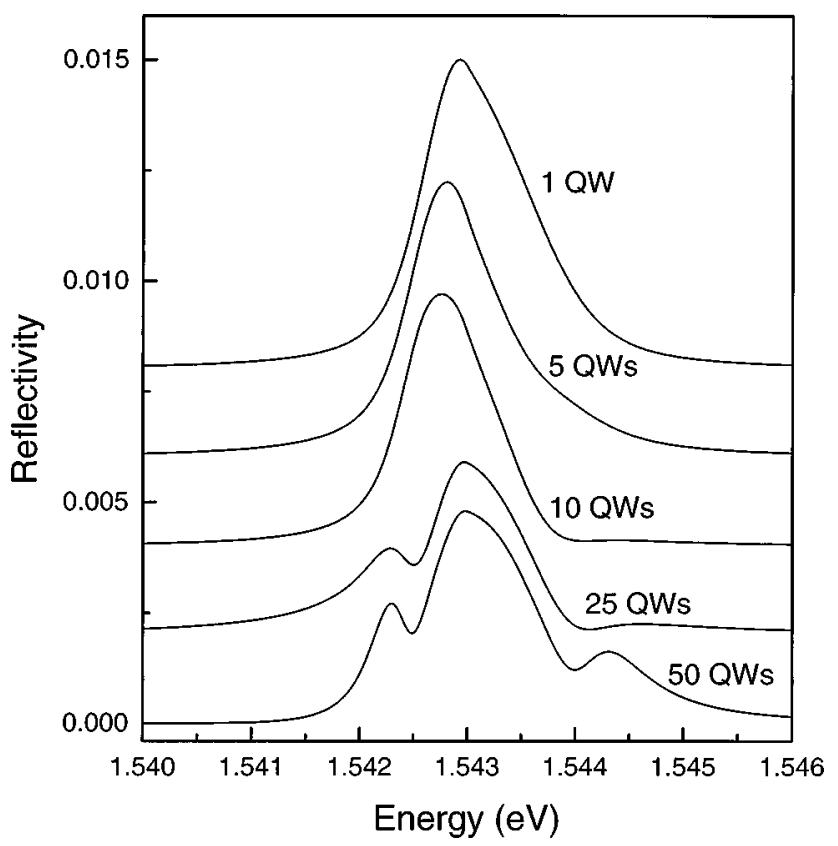

FIG. 3. Reflectivity spectra of periodic structures containing 1, 5, 10, 25, and $50 \mathrm{QW}$ 's. For all structures, $\hbar \omega_{0}=1.543 \mathrm{eV}, d$ $=240 \AA, \gamma=0, \hbar \Gamma_{0}=0.026 \mathrm{meV}, \Delta_{1}=0.4 \mathrm{meV}$, and $\Delta_{2}=0.7 \mathrm{meV}$.

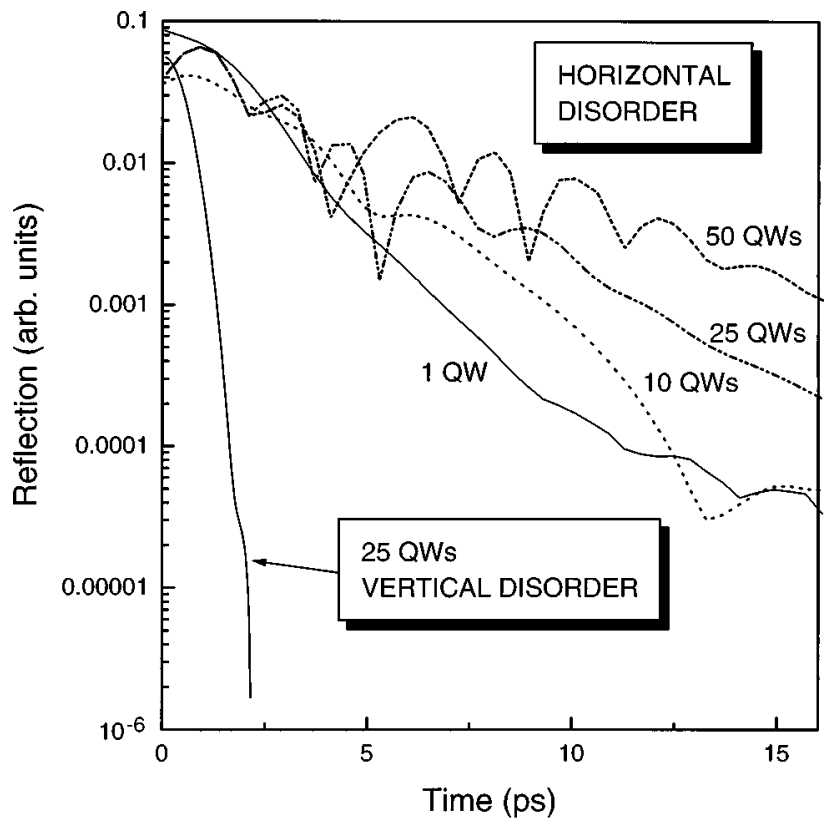

FIG. 4. Time-resolved reflection dynamics for a $\delta$ pulse of light incident on the periodic structures containing $1,10,25$, and 50 QW's with the same parameters as in Fig. 3. Also shown is the reemission from a vertically disordered MQW structure containing 25 QW's, where the exciton resonance energy in each well exceeds by $0.02 \mathrm{meV}$ that in the underlying well.

tended exciton-polariton states in $10 \mathrm{QW}$ 's better average the disorder than the polariton modes in 5 QW's and indeed better than the lone exciton-polariton state in the single QW. From Fig. 2 we see that for $N=5$ or 10 the amount of disorder assumed strongly suppresses the oscillator strength of bright modes. In this regime, we cannot see a fine structure connected with different polariton modes (they are too weak). The role of polaritons consists in vertical averaging of the disorder potential.

The transition to another regime that can be called "superradiant" occurs at $N \sim 25$. In this case two distinct peaks are now seen in the spectrum. They correspond to two bright modes having sufficient oscillator strength to be seen in spite of the inhomogeneous broadening. The total linewidth increases with respect to the $10 \mathrm{QW}$ structure, because of the substantial splitting between the two superradiant modes. In the $50 \mathrm{QW}$ structure one can already see three peaks corresponding to three modes bright enough to resist disorder. The linewidth in this case is, of course, wider.

Thus, searching for optimum samples with extremely narrow exciton lines, one should try to avoid the limits of single QW structures and structures with a huge number of QW's (e.g., 50). For the $\mathrm{GaAs} / \mathrm{Al}_{x} \mathrm{Ga}_{1-x} \mathrm{As}$ system considered here, the narrowest lines have been found for $N \sim 10$ provided that the potential fluctuations have the same amplitude in all the wells in all the model structures.

Time-resolved transmission or reflection spectra may give much additional information about exciton polaritons and disorder in MQW's. Superradiance theory that ignores the disorder predicts the decrease of the exciton radiative lifetime with increasing number of QW's in the structure, since the radiative lifetime is inversely proportional to the imaginary part of the polariton eigenenergy. In the presence of 
disorder this tendency is reversed. Figure 4 shows the calculated time-resolved reflection spectra of the same MQW structures as before, for the case of an incident ultrashort $(\delta)$ pulse of light. In this case, the time-resolved reflection is simply described by a Fourier transform of the complex amplitude reflection coefficient. If all the QW's are equivalent and experience in-plane potential fluctuations (horizontal disorder case) the signal decays slower with increasing number of QW's. For all cases, the decay time is shorter both than that expected in the situation of no disorder and that detected in record quality $\mathrm{GaAs} / \mathrm{Al}_{x} \mathrm{Ga}_{1-x} \mathrm{As} \mathrm{MQW}$ 's i.e., about 12 ps (Ref. 16 and the references cited therein). Clearly, the inhomogeneous broadening causes exciton coherences to decay much faster, while increasing the number of QW's reduces the effect of the disorder, so that the exciton lifetime increases. The appearance of distinct superradiant modes in 25 and $50 \mathrm{QW}$ structures leads to pronounced oscillations in time-resolved spectra caused by quantum beats between these modes. Note that in these non-Braggarranged structures, the long time decay rates are still slower for larger numbers of QW's, and thus are not truly superradiant decays.

Both motional narrowing and superradiance effects require the coherence between excitons localized in different QW's. This coherence can be broken if one introduces vertical disorder, i.e., differences between exciton resonance frequencies $\left(\omega_{0}\right)$ in different QW's. Figure 4 shows the time-resolved reflection dynamics calculated for the same 25 QW structure as earlier but with exciton resonance frequencies in the QW's equidistantly distributed between 1543.0 and $1543.5 \mathrm{meV}$. One can see that the decay of the signal is much faster in this case than in that with only horizontal disorder, signaling the disappearance of the motional narrowing effect. Moreover, the oscillations of the signal are smeared out, thus no superradiant modes exist in the structure.

In conclusion, semiclassical treatment of the exciton polaritons in MQW's in the presence of in-plane potential fluctuations reveals pronounced motional narrowing and superradiance effects. The motional narrowing regime is realized when the inhomogeneous broadening much exceeds the radiative width of bright exciton-polariton modes in the structure. This reflects the change in the size of the quantum state responsible for coupling light into the structure, and manifests itself in the decrease of the linewidth of the exciton resonance in reflection and the increase of the exciton radiative lifetime, with increasing number of QW's. The optical interference between different QW's is responsible for the vertical averaging of potential fluctuations in MQW structures. This is fundamentally different from the earlier reported horizontal motional narrowing effect due to averaging of disorder in the plane of a quantum microcavity. The superradiance effects dominate when the radiative decay rate of the bright modes exceeds the inhomogeneous broadening. In this regime, pronounced peaks corresponding to superradiant polariton modes are seen in the $\mathrm{cw}$ reflection spectra while time-resolved reflection spectra show oscillations originating from the beats between these modes. Vertical disorder suppresses both motional narrowing and superradiance effects.

We are deeply grateful to Lucio Claudio Andreani, Jeremy Allam, and Albert Heberle for many useful discussions.
*Permanent address: A. F. Ioffe Physico-Technical Institute, 26 Politechnicheskaya, 194021, St. Petersburg, Russia.

${ }^{1}$ V. M. Agranovich and O. A. Dubovskii, Zh. Eksp. Teor. Fiz. Pis'ma Red. 3, 345 (1966) [JETP Lett. 3, 345 (1966)].

${ }^{2}$ See, for example, A. Abragam, The Principles of Nuclear Magnetism (Clarendon, Oxford, 1961), p. 446.

${ }^{3}$ D. S. Citrin, Solid State Commun. 89, 139 (1994); G. Björk, S. Pau, J. M. Jakobson, H. Cao, and Y. Yamamoto, Phys. Rev. B 52, 17310 (1995).

${ }^{4}$ See, e.g., papers in Proceedings of the Fourth International Meeting on Optics of Excitons in Confined Systems, City, Country, edited by Editors [Nuovo Cimento D 17 (1996)].

${ }^{5}$ T. Stroucken, A. Knorr, C. Anthony, A. Schulze, P. Thomas, S. W. Koch, M. Koch, S. T. Cundiff, J. Feldmann, and E. O. Göbel, Phys. Rev. Lett. 74, 2391 (1995).

${ }^{6}$ R. Zimmermann, Proceedings of the Fourth International Meeting on Optics of Excitons in Confined Systems, City, Country, edited by Editors [Nuovo Cimento D 17, 1801 (1996)].

${ }^{7}$ D. M. Whittaker, P. Kinsler, T. A. Fisher, M. S. Skolnick, A.
Armitage. A. M. Afshar, M. D. Sturge, and J. S. Roberts, Phys. Rev. Lett. 77, 4792 (1996).

${ }^{8}$ V. Savona, C. Piermarocchi, A. Quattropani, F. Tassone, and P. Schwendimann, Phys. Rev. Lett. 78, 4470 (1997).

${ }^{9}$ A. V. Kavokin, Phys. Rev. B 57, 3757 (1998).

${ }^{10}$ J. J. Baumberg, A. P. Heberle, A. V. Kavokin, M. R. Vladimirova, and K. Köhler, Phy. Rev. Lett. 80, 3567 (1998).

${ }^{11}$ L. C. Andreani, G. Panzarini, A. V. Kavokin, and M. R. Vladimirova, Phys. Rev. B 57, 4670 (1998).

${ }^{12}$ D. M. Whittaker, cond-mat/9711290 (unpublished).

${ }^{13}$ D. S. Citrin, Phys. Rev. B 47, 3832 (1993).

${ }^{14}$ M. R. Vladimirova, E. L. Ivchenko, and A. V. Kavokin, Semiconductors 32, 90 (1998).

${ }^{15}$ E. L. Ivchenko, S. Jorda, and A. I. Nesvizhskii, Superlattices Microstruct. 16, 17 (1996); M. Hübner, J. Kuhl, T. Stroucken, A. Knorr, S. W. Koch, R. Hey, and K. Ploog, Phys. Rev. Lett. 76, 4199 (1996).

${ }^{16}$ B. Deveaud, F. Clerot, N. Roy, S. Satzke, B. Sermage, and D. S. Katzer, Phys. Rev. Lett. 67, 2355 (1991). 